\title{
SYNTHESIS OF SINGLE CRYSTALLINE TITANIUM OXIDE AND SODIUM TITANATE NANORODS VIA SALT-ASSISTED ULTRASONIC SPRAY PYROLYSIS
}

\begin{abstract}
The simple and continuous synthesis of single crystalline anatase titanium dioxide and sodium titanate nanorods by a saltassisted ultrasonic spray pyrolysis method is demonstrated. This method does not require expensive precursors, long reaction time, and physical templates or surfactant. In addition, its continuous nature makes it a suitable method for the large-scale preparation. Moreover, the effect of a salt concentration in a starting solution on material properties, including morphology and phase of the synthesized products was systematically investigated. The synthesized nanorods had one-dimensionality, a single crystalline and the average diameter of $12.3 \mathrm{~nm}$ with dual phases of titanium dioxide and sodium titanate by FE-SEM, XRD, HR-TEM as well as FFT-converted SAED pattern analysis.
\end{abstract}

Keywords: $\mathrm{TiO}_{2}$, Nanorods, Salt, Ultrasonic Spray Pyrolysis, Optical properties

\section{Introduction}

As one of the most important metal oxides and semiconductors, titanium dioxide $\left(\mathrm{TiO}_{2}\right)$, especially anatase and rutile $\mathrm{TiO}_{2}$, is the most extensively studied material. It is known to be a very useful non-toxic, environmental friendly, and corrosionresistant material with unique ionic and electronic properties that enable its use in solar cells, photocatalytic reactions, and lithium-ion batteries [1-4]. Generally, the performance of these applications is mainly determined by a surface reaction/interaction and a charge transfer or separation. Therefore, it is crucial to maximize the specific surface area and control the morphology to achieve a maximum overall efficiency. From this point of view, nanoparticulated forms of $\mathrm{TiO}_{2}$ with one-dimensional (1D) structures including nanorods, nanowires, nanotubes and nanofibers are the most suitable because they provide a higher specific surface area and electron mobility compared with other nanosize geometries [5-7].

Various methods have been reported on the synthesis of $1 \mathrm{D} \mathrm{TiO}_{2}$ nanostructures including sol-gel methods, hydro/solvothermal approaches, template-assisted methods and by electrochemical means [8-10]. These current synthesis routes require expensive precursors or templates and complicated or multi-step procedures, moreover, all of them are batch type processes which require a long reaction time and thus, the processes are not compatible for a cost-effective mass production. In addition, it is difficult to obtain single crystalline $1 \mathrm{D} \mathrm{TiO}{ }_{2}$ nanostructures via the methods because the common titanium precursors for the solution-based methods exhibit a hydrolytic reaction rates which make it difficult to control their reaction processes and invariably lead to a mixture of different polymeric species [11-12].

Given its simple procedure and continuous nature, ultrasonic spray pyrolysis (USP) is an attractive method for the large-scale preparation of materials, but polycrystalline spheres with the size of a sub-micron or few micron meter are typically produced because there is a limitation to reduce an initial droplet size through an ultrasound. Recently, controlling their size, morphology and crystalline nature has been achieved by the incorporation or in situ generation of inert or reactive salts such as $\mathrm{NaCl}, \mathrm{NaNO}_{3}$ or $\mathrm{KNO}_{3}$ during USP [13-15]. However, relatively little research has been carried out on nanoscale materials with both 1D structure and single-crystalline nature. Furthermore, to the best of our knowledge, this is the first time to apply this technique in the synthesis of single crystalline $\mathrm{TiO}_{2}$ nanorods. In this study, we demonstrated that salt-assisted ultrasonic spray pyrolysis (SA-USP) could be used as an alternative process to synthesize single crystalline $\mathrm{TiO}_{2}$ nanorods. The effect of salt contents in precursor solutions on the formation of $\mathrm{TiO}_{2}$ particles was systematically investigated. In addition, the crystallography and microstructure of the synthesized nanostructure were also investigated and discussed.

\section{Experimental}

All the reagents and solvent were purchased from Aldrich and used directly without further purification. Starting solutions for the SA-USP were prepared by mixing hexafluorotitanic acid solution $\left(\mathrm{H}_{2} \mathrm{TiF}_{6}, 60 \mathrm{wt} . \%\right.$ in $\mathrm{H}_{2} \mathrm{O}$, Sigma aldrich $)$ and dissolving

\footnotetext{
* DEPT. OF MATERIALS SCIENCE AND ENGINEERING, SEOUL NATIONAL UNIVERSITY OF SCIENCE AND TECHNOLOGY, SEOUL-SI 01811, REPUBLIC OF KOREA 
sodium chloride $(\mathrm{NaCl}, 99.5 \%)$ in in distilled water. The concentration of the $\mathrm{H}_{2} \mathrm{TiF}_{6}$ was fixed to $40 \mathrm{mM}$ but the $\mathrm{NaCl}$ content was varied to $40,200,300$ and $400 \mathrm{mM}$. The molar ratios of the $\mathrm{NaCl}$ were $1,5,7.5$ and 10 of the $\mathrm{H}_{2} \mathrm{TiF}_{6}$. Hereafter, the samples were named T1, T5, T7.5 and T10 to reflect the relative content of the slat. The prepared precursor solutions were ultrasonically nebulized at a frequency of $1.7 \mathrm{MHz}$ into microdroplets, which carried by a gas flow into a tube furnace where solvent evaporation and precursor decomposition occurred, for producing a $\mathrm{NaCl} / \mathrm{TiO}_{2}$ composite powder. The furnace with a quartz tube (30 $\mathrm{mm}$ inner diameter and $500 \mathrm{~mm}$ long) was preheated to $800^{\circ} \mathrm{C}$, and compressed pure oxygen was used as carrier gas at a flow rate of $2 \mathrm{~L} / \mathrm{min}$. The $\mathrm{NaCl} / \mathrm{TiO}_{2}$ powder was collected by the incorporation of a filter paper (Qualitative Filter Paper, 5-8 $\mu \mathrm{m}$, Hyundai Micro Co., Ltd., Korea) in the carrier gas stream and then was washed with distilled water several times using a probetype ultrasonicator (VC-505, Sonics \& Materials, Inc, USA) at 300 watts to dissolve and remove the $\mathrm{NaCl}$ from the powder.

The crystal structures and crystallite size of the $\mathrm{NaCl} / \mathrm{TiO}_{2}$ composite powder and $\mathrm{TiO}_{2}$ nanoparticles were characterized by X-ray diffractometer (XRD, X'Pert ${ }^{3}$ Powder, PANalytical, Netherlands) with a radiation wavelength of $\lambda=1.54056^{\circ} \mathrm{A}$. XRD patterns were obtained from $20^{\circ}$ to $80^{\circ}$ of $2 \mathrm{q}$ at a scanning rate of $4^{\circ} \mathrm{min}^{-1}$. Microstructural characterization of the as-synthesized and washed powder was performed by means of field emission scanning electron microscopy (FE-SEM, JSM-7500F, JEOL, Japan) and high-resolution transmission electron microscopy (HRTEM, JEM-2100F, JEOL, Japan). An absorption spectrum was measured in the region of $200-900 \mathrm{~nm}$ with an ultraviolet/ visible (UV/Vis) spectrophotometer (UV-vis DRS, UV-2600, SHIMADZU, Japan).

\section{Results and discussion}

Figure 1 shows the FE-SEM images of as-synthesized and washed powders with respect to the ratio of the added salt in the precursor solution (T1, T5, T7.5 and T10). Initial droplets nebulized by ultrasound are spherical generally due to a surface tension. A gas then carries the droplets into a furnace, with many possible reaction pathways which determine powder morphologies including solid, hollow or fragmented products. As shown in Fig. 1, all as-prepared powders $(\mathrm{A} \sim \mathrm{D})$ are solid, but their morphologies are quite different. The T1 powder with a low $\mathrm{NaCl}$ content shows near spherical morphologies, however, the morphology slightly changes to a cube which is the crystal structure of $\mathrm{NaCl}$ with increasing the $\mathrm{NaCl}$ content. All powders show rough surfaces because they are secondary particles consisted of tiny primary nanoparticles. The $\mathrm{NaCl}$ acts as a high temperature solvent that prevents the hard agglomeration and densification of the primary nanoparticles. If the concentration of the $\mathrm{NaCl}$ is high enough, the secondary powder disintegrated to the primary particles by washing the $\mathrm{NaCl}$. The morphologies of the washed powders strongly depends on the $\mathrm{NaCl}$ contents as shown in Fig. 1E-H. After the $\mathrm{NaCl}$ removal, the $\mathrm{T} 1$ powder is a spherical shape with some pores and the T5 one is in the form of skin, whereas T7.5 and T10 samples are separated to tiny primary particles which have the desired sizes and morphologies.

The phase of intermediate product after the SA-USP was analyzed by XRD, as shown in Fig. 2. The diffraction peaks of cubic $\mathrm{NaCl}$ (JCPDS No. 05-6278) were observed from all samples. However, except for the T1 powder, the others do not show the diffraction pattern of anatase $\mathrm{TiO}_{2}$ (JCPDS No. 21-1272) despite the sufficient quantity of the titanium precursor in the starting solution. On the basis of the morphology change before and after removing the $\mathrm{NaCl}$ shown in Fig. 1, this can be interpreted that tiny $\mathrm{TiO}_{2}$ primary particles are well dispersed in the $\mathrm{NaCl}$ matrix or form very thin shells, thus they produce extremely broaden diffraction peaks with low intensities compared to those of $\mathrm{NaCl}$. The presence of $\mathrm{TiO}_{2}$ was confirmed from the XRD patterns after washing the powders as shown in Fig. 3.

The $\mathrm{NaCl}$ diffraction peaks were completely disappeared except for the T1 powder and all samples indicated the diffraction patterns corresponded to anatase $\mathrm{TiO}_{2}$. However, the powder
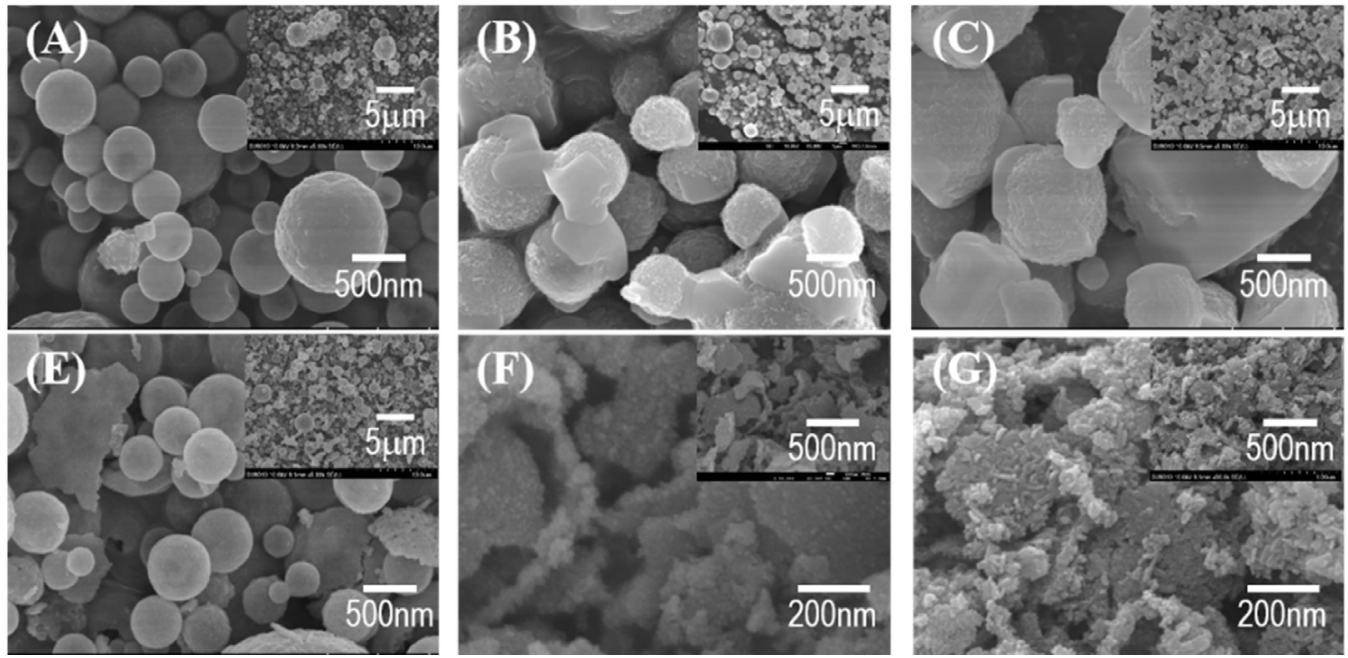

Fig. 1. FE-SEM images of (A-D) as-synthesized and (E-F) washed powders with respect to the ratio of the added salt in the precursor solution; (A,D) T1, (B,E) T5, (C,G) T7.5 and (D,H) T10 


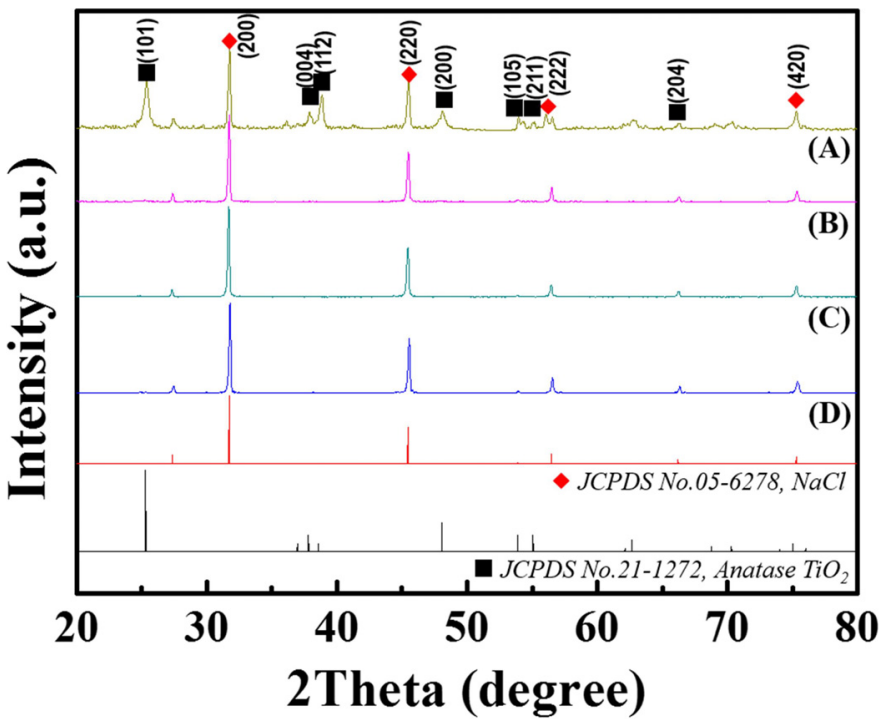

Fig. 2. XRD patterns of the as-prepared powders by SA-USP at $800^{\circ} \mathrm{C}$ as a function of the salt content; (A) T1, (B) T5, (C) T7.5 and (D) T10

synthesized from the starting solution with relatively high $\mathrm{NaCl}$ concentrations (sample T5, T7.5 and T10) showed the diffraction peaks of monoclinic sodium titanate $\left(\mathrm{Na}_{2} \mathrm{Ti}_{6} \mathrm{O}_{13}\right.$, JCPDS No. 37-0951). This phase is known to be one of the ternary M-Ti-O $(\mathrm{M}=$ alkali metal) system materials which have low toxicity, low cost and wide applications including sodium ion batteries and photocatalysts [16-19]. Although the synthesized nanostructures were not pure $\mathrm{TiO}_{2}$ but the $\mathrm{Na}_{2} \mathrm{Ti}_{6} \mathrm{O}_{13}$ is also useful and the properties and applications of $\mathrm{Na}_{2} \mathrm{Ti}_{6} \mathrm{O}_{13}$ are quite similar to anatase $\mathrm{TiO}_{2}$. In addition, they could be selectively synthesized by modulating the pyrolysis condition such as temperature and gas flow rate. Analysis of the reflection at the diffraction peaks

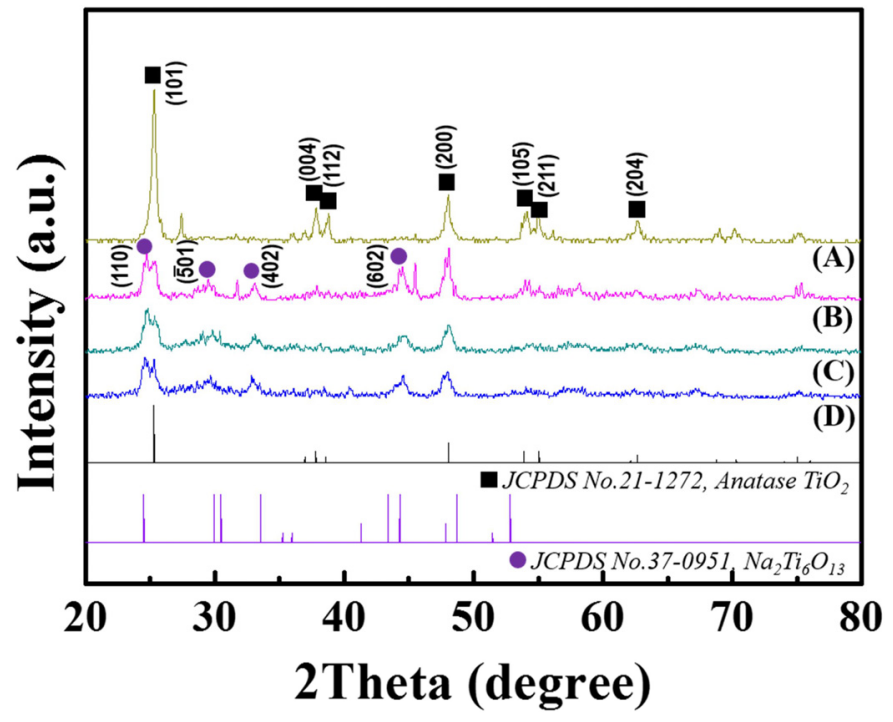

Fig. 3. XRD patterns of the powders after removing the $\mathrm{NaCl}$; (A) T1, (B) T5, (C) T7.5 and (D) T10

of (101) and (200) for the T7.5 powder by the Scherrer equation [20] revealed that an average crystallite size is $16 \mathrm{~nm}$ and $16.5 \mathrm{~nm}$, respectively.

The crystallography and microstructure of the synthesized $\mathrm{TiO}_{2}$ and $\mathrm{Na}_{2} \mathrm{Ti}_{6} \mathrm{O}_{13}$ nanostructures were further studied using TEM, high-resolution (HR) TEM and fast Fourier transform (FFT) analysis. Fig. 4A is a low magnification TEM image of the T7.5 powder synthesized by the SA-USP at $800^{\circ} \mathrm{C}$ and the washing treatment. It can be seen from the image that the nanostructures are longish in shape with a small aspect ratio. The average diameter was determined by using Image $\mathbf{J}$ software and the value was about $12.3 \mathrm{~nm}$ that was in good agreement
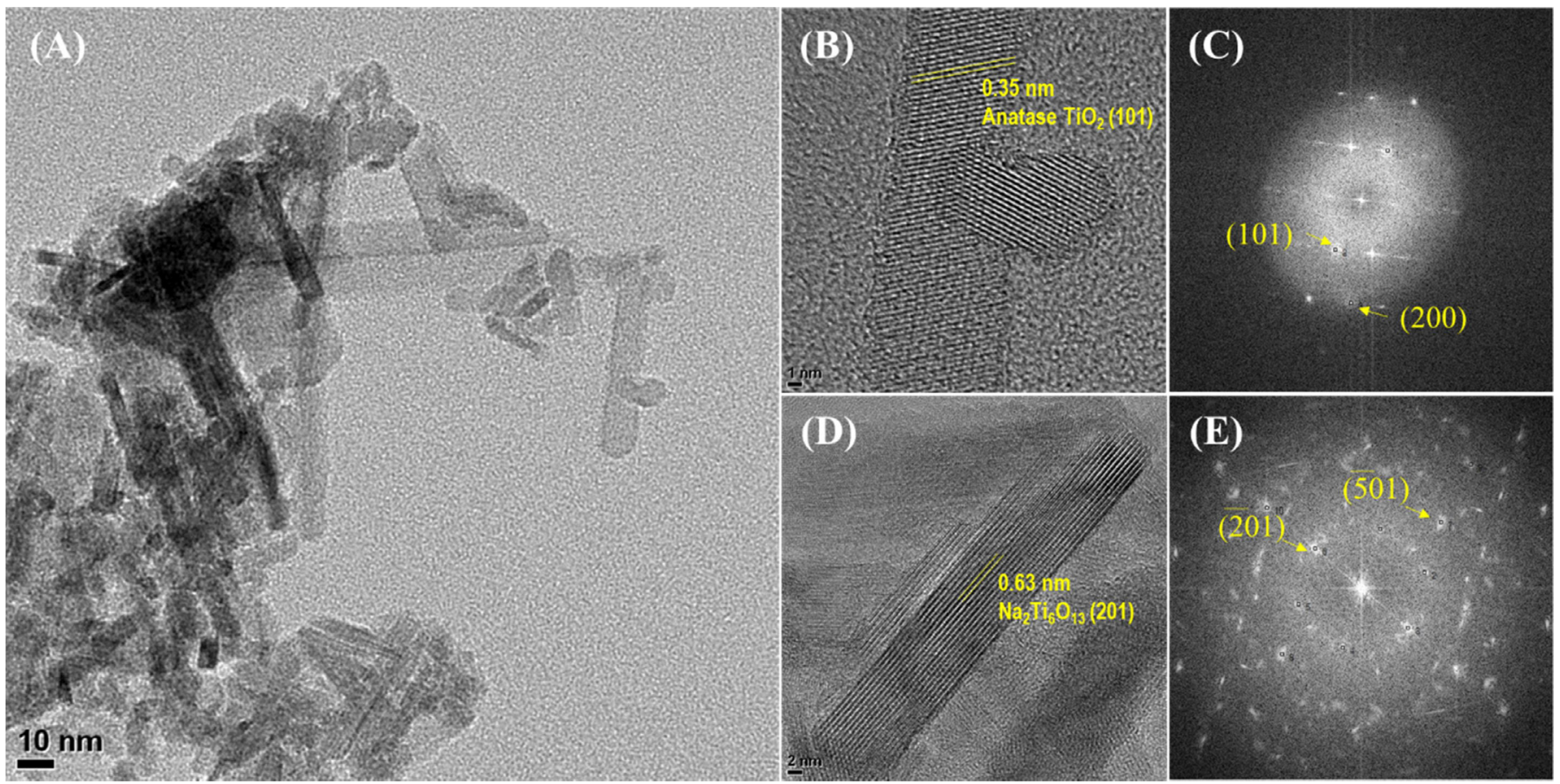

Fig. 4. Low-magnification TEM, high resolution TEM and fast Fourier transform-converted selected area electron diffraction patterns of the T7.5 powder 
with the crystallite size calculated by Scherrer equation from the XRD pattern. As shown in Fig. 4B,D, the HR-TEM images of randomly selected nanorods show that the individual nanorods are structurally single-crystalline in nature and have a periodic fringe spacing of $0.35 \mathrm{~nm}$ and $0.63 \mathrm{~nm}$ along their longitudinal or transverse axes, which correspond to the interplanar spacing between the (101) planes of anatase $\mathrm{TiO}_{2}$ and the (201) planes of monoclinic $\mathrm{Na}_{2} \mathrm{Ti}_{6} \mathrm{O}_{13}$, respectively. The corresponding FFTconverted selected area electron diffraction (SAED) patterns obtained from the HR-TEM images indicated that the nanorods had single-crystalline structures. The patterns shown in Fig. 4C,E were consistent with the d-spacing value of the (101), (200) and (201), (501) lattice planes of anatase $\mathrm{TiO}_{2}$ and monoclinic $\mathrm{Na}_{2} \mathrm{Ti}_{6} \mathrm{O}_{13}$ with corresponding d-spacings of $0.352,0.189 \mathrm{~nm}$ and 0.629 and $0.298 \mathrm{~nm}$, respectively. This was consistent with the results obtained from the XRD analysis.

\section{Conclusions}

In summary, we have demonstrated a simple and continuous synthesis method for synthesizing single-crystalline nanorods using a $\mathrm{NaCl}$-assisted ultrasonic spray pyrolysis followed by the removal treatment of the $\mathrm{NaCl}$. The concentration of the $\mathrm{NaCl}$ in starting precursor solutions play an important role to determine the size and morphology of the synthesized powders. The powder prepared from the precursor solution with the $\mathrm{Ti} / \mathrm{Na}$ molar ratio of $1: 7.5$ was composed of single crystalline nanorods with the average diameter of $12.3 \mathrm{~nm}$. The nanorods have dual phases being anatase $\mathrm{TiO}_{2}$ and monoclinic $\mathrm{Na}_{2} \mathrm{Ti}_{6} \mathrm{O}_{13}$ which is a reaction phase with $\mathrm{Na}$ and $\mathrm{TiO}_{2}$. However, the phases could be readily controlled by changing the pyrolysis condition. These materials could have a wide range of applications in photocatalysts, solar cells and lithium ion batteries because of their large specific surface areas, dimensionalities and high crystallinities.

\section{Acknowledgments}

This work was supported by the materials and parts technology development program (grant no. 10049555) funded by the Korean Government Ministry of Trade, Industry \& Energy and Basic Science Research Program through the National Research Foundation of Korea (NRF) funded by the Ministry of Science, ICT and Future Planning (grant no. NRF2015R1C1A1A02037745).

\section{REFERENCES}

[1] H. Melhem, P. Simon, L. Beouch, F. Goubard, M. Boucharef, C.D. Bin, Y. Leconte, B. Ratier, N. Herlin-Boime, J. Boucle, Adv. Energy Mater. 1, 908 (2011).

[2] J. Huo, Y. Hu, H. Jiang, W. Huang, Y. Li, W. Shao, C. Li, Ind. Eng. Chem. Res. 52, 11029 (2013).

[3] M.D. Hernandez-Alonso, F. Fresno, S. Suarez, J.M. Coronado, Energy Environ. Sci. 2, 1231 (2009).

[4] K. Zhu, X. Liu, J. Du, J. Tian, Y. Wang, S. Liu, Z. Shan, J. Mater. Chem. A. 3, 6455 (2015).

[5] H. Wang, M. Liu, M. Zhang, P. Wang, H. Miura, Y. Cheng, J. Bell, Phys. Chem. Chem. Phys. 13, 17359 (2011).

[6] J. Cai, J. Ye, S. Chen, X. Zhao, D. Zhang, S. Chen, Y. Ma, S. Jin, L. Qi, Energy Environ. Sci. 5, 7575 (2012).

[7] Y. Guo, G. Liu, Z. Ren, A. Piyadasa, P.X. Gao, CrystEngComm. 15, 8345 (2013).

[8] D.H. Kim, W.M. Seong, I.J. Park, E.S. Yoo, S.S. Shin, J.S. Kim, H.S. Jung, S.W. Lee, K.S. Hong, Nanoscale. 5, 11725 (2013).

[9] M. Ge, C. Cao, J. Huang, S. Li, Z. Chen, K.Q. Zhang, S.S. Al-Deyab, Y. Lai, J. Mater. Chem. A. 4, 6772 (2016).

[10] W.J. Ong, L.L. Tan, S.P. Chai, S.T. Yong, A.R. Mohamed, Nanoscale. 6, 1946 (2014).

[11] S.S. Chen, X. Mao, Chem. Rev. 107, 2891 (2007).

[12] M. Liu, L. Piao, L. Zhao, S. Ju, Z. Yan, T. He, C. Zhou, W. Wang, Chem. Commun. 46, 1664 (2010).

[13] G.H. An, T.Y. Hwang, J.R. Kim, J.B. Kim, N.S. Kang, S.I. Kim, Y.M. Choi, Y.H. Choa, J. Alloy. Compd. 583, 145 (2014).

[14] Y. Itoh, I.W. Lenggoro, J. Mater. Res. 17, 3222 (2002).

[15] N. Shimizu, K. Myoujin, T. Kodera, T. Ogihara, Key Eng. Mater. 566, 281 (2013).

[16] Y. Zhang, H. Hou, X. Yang, J. Chen, M. Jing, Z. Wu, X. Jia, X. Ji, J. Power Sources. 305, 200 (2016).

[17] W. Wang, C. Yu, Z. Lin, J. Hou, H. Zhu, S. Jiao, Nanoscale. 5, 594 (2013).

[18] Z. Zhang, J.B.M. Goodall, S. Brown, L. Karlsson, R.J.H. Clark, J.L. Hutchison, I.U. Rehman, J.A. Darr, Dalton Trans. 39, 711 (2010).

[19] C.Y. Xu, J. Wu, P. Zhang, S.P. Hu, J.X. Cui, Z.Q. Wang, Y.D. Huang, L. Zhen, Cryst. Eng. Comm. 15, 3448 (2013).

[20] A.L. Patterson, Phys. Rev. 56, 978 (1939). 